\title{
Infarto cerebral en recién nacidos a término con convulsiones
}

\author{
Dr. Marcelo Devilat B. ${ }^{1}$; Dr. Roger Lamas G. ${ }^{2}$; Dr. Mario Castro A. ${ }^{3}$ \\ Cerebral infarction in full-term \\ newborns with seizures
}

\begin{abstract}
Among 13 fuli-term newborn jnfants with seizures in the first week of life, that were discharged from a neonathologic unit from July 1983 to December 1986, cerebral infarction was the second most frequent cause of fits (3/13), after the unknown. This three patients are presented: in two the ethiology of cerebral infarctions was not identified, in the third one it was related to hipovolemic shock and acidosis. Clinical neurological examination and anterior fontanelle ultrasonography do not seem to be sensitive enough to detect the infarction but focal convulsive fits and electroencephalogram may suggest the diagnosis. Definite diagnosis requires computed axial tomography. Follow up for one to two years shows spatic hemiparesia in one patient, mixed epilepsy in another one and febrile convulsions in the third one, without other evidence of psycomotor retardation.
\end{abstract}

(Key words: newborn infants, convulsions, cerebral infarction.)

Las convulsiones neonatales son la alteración neurológica más frecuente en el recién nacido ${ }^{1} \mathrm{y}$ sus causas obedecen a diversos factores ${ }^{2},{ }^{3}$. Entre ellas, el infarto cerebral no ha sido lo suficientemente destacado, a pesar de ser común en necropsias de recién nacidos de término (RNT), y en RNT con convulsiones egresados viyos en las unidades de neonatología ${ }^{4}, 5$. Hasta marzo de 1986 se habian comunicado en la literatura 42 casos de recién nacidos vivos con infarto cerebral, en su mayoría RNT con convulsiones $s^{4}, 6-13$.

El propósito de esta comunicación es presentar a 3 RNT con convulsiones e infarto cerebral, analizar la etiología del cuadro en estos pacientes, discutir los elementos del diagnóstico e informar acerca de la evolución posterior.

\section{CASOS CLINICOS}

En la tabla 1 se observan las características de los pacientes. Todos eran RNT adecuados a su edad gestacional. El peso de nacimiento fluctuó entre $2.670 \mathrm{~g} \mathrm{y}$ $3.400 \mathrm{~g}$. Ninguno tenía antecedentes familiares de epilepsia. Todos los pacientes tenían glicemia, calcemia, hemograma, VDRL y electrolitos plasmáticos nomales;

1. Unidad de Neurología, Servicio de Pediatría. Hospital Militar. Facultad de Medicina de la Universidad de Chile.

2. Servicio de Neonatología, Hospital Militar.

3. Servicio de Neurorradiología, Hospital Militar. uno de ellos eursó con hipovolemia y acidosis por sangramiento fetoplacentario.

En la tabla 2 se aprecia la evolución de los enfermos, controlados entre 1 año y 2 años 7 meses; uno muestra déficit notor caractetizado por hemiparesia espástica unilaterai; los otros dos han suftida respectivamente epilepsia y convalsiones febriles, pero ninguno muestra alteraciones significativas del desarrollo psicomotor.

\section{COMENTARIO}

Los 3 pacientes tienen algunas características comunes: son RNT con convulsiones, Apgar normal, sin epilepsia entre sus familiares, hipotonía al examen físico, liquido cefalorraquídeo (LCR) hemorrágico e infarto cerebral demostrado en la tomografía axial computada (TAC). Este cuadro, que pareciera ocupar un lugar importante dentro de las convulsiones neonatales ${ }^{4}$, no ha sido suficientemente difundido. Algunos autores to han mencionado ${ }^{2}$, sin informar acerca de su frecuencia, o bien, asociándola a estados de Mal epiléptico focal en recién nacidos ${ }^{14}$; otros, en cambio, lo ignoran ${ }^{1,3,15}$. Es posible que dentro de las llamadas convulsiones neonatales benignas ${ }^{\mathrm{j}, 16}$ exista cierto número cuyo origen pudiera ser debido a infartos cerebrales idiopáticos, que ocurrieron, aparentemente, en 2 de nuestros 3 pacientes, hecho que también ha sido destacado por otros ${ }^{4,11}$. 
Tabla 1

Características de los pacientes con infarto cerebral

\begin{tabular}{|c|c|c|c|}
\hline Paciente & 1 & 2 & 3 \\
\hline $\begin{array}{l}\text { Antecedentes pre, } \\
\text { peri y postnatales. }\end{array}$ & Normales. & $\begin{array}{l}\text { Sufrimiento fetal } \\
\text { prenatal - acidosis. } \\
\text { Shock hípovolémico. }\end{array}$ & Notmales. \\
\hline Apgar 1 y $5 \mathrm{~min}$. & 8 y 9 & $7 \times 8$ & 8 y 9 \\
\hline Tipo de crisis. & Motora izquierda. & Tónica generalizada. & Tónico-clónica derecha. \\
\hline Examen & $\begin{array}{l}\text { Hipotonía } \\
\text { grito. }\end{array}$ & $\begin{array}{c}\text { Hipotonia } \\
\text { hipoactividad. }\end{array}$ & $\begin{array}{c}\text { Hipotonía } \\
\text { hipoactividad. }\end{array}$ \\
\hline Gases del cordón. & Normales. & $\begin{array}{c}\text { Acidosis metabólica } \\
\text { compensada. }\end{array}$ & Normales. \\
\hline TORCH & Normales. & $N / R$ & Normales. \\
\hline $\begin{array}{l}\text { Aminoácjdos en } \\
\text { sangre y orina. }\end{array}$ & $N / R$ & Normales. & $N / R$ \\
\hline $\begin{array}{l}\text { Edad de las } \\
\text { crisis: dias }\end{array}$ & 2 & 1 & 2 \\
\hline $\begin{array}{c}\text { EEG: edad (días). } \\
\text { : Resultado. }\end{array}$ & $\begin{array}{c}3 \\
\text { Normal. }\end{array}$ & $\begin{array}{l}10 \\
\text { Focal derecho. }\end{array}$ & $\begin{array}{c}7 \\
\text { Focal izquierdo. }\end{array}$ \\
\hline $\begin{array}{c}\text { ECO: edad (días). } \\
\text { : Resultado. }\end{array}$ & $\begin{array}{c}3 \\
\text { Normal. }\end{array}$ & $\begin{array}{c}2 \\
\text { Normal. }\end{array}$ & $\begin{array}{c}3 \text { y } 7 \\
\text { Normal. }\end{array}$ \\
\hline TAC: edad (dias). & $\begin{array}{c}2 \\
\text { Infarto derecho. }\end{array}$ & $\begin{array}{l}2 \\
\text { Hemorragia subaracroidea } \\
\text { bilateral infarto derecho. }\end{array}$ & $\begin{array}{c}7 \\
\text { Infarto izquierdo. }\end{array}$ \\
\hline Territotio arterial. & $\begin{array}{c}\text { Cerebtal anterior } \\
\text { derecha. }\end{array}$ & $\begin{array}{l}\text { Cerebral media } \\
\text { derecha. }\end{array}$ & $\begin{array}{l}\text { Cerebral media } \\
\text { izquierda. }\end{array}$ \\
\hline
\end{tabular}

$\mathrm{N} / \mathrm{R} \approx$ examen no analizado.

$\mathrm{EEG}$ = electroencefalograma. $\mathrm{ECO}=$ ecograf ía encefálica. TAC = tomografía axial computada. TORCH $=$ pruebas serológicas para toxoplasma, virus rubéola y tripanosoniasis (Chagas).

Tabla 2

Erolución de pacientes con infarto cerebral

\begin{tabular}{cccc}
$\begin{array}{c}N^{0} \\
\text { paciente }\end{array}$ & $\begin{array}{c}\text { Tiempo } \\
\text { en control }\end{array}$ & $\begin{array}{c}\text { Crisis } \\
\text { epilépticas }\end{array}$ & Convulsión febril. \\
1 & 2 años $7 \mathrm{~m}$ & - & $\begin{array}{c}\text { Examen neurológico y } \\
\text { DSM }\end{array}$ \\
3 & 1 año $8 \mathrm{~m}$ & Epilepsia. & $\begin{array}{c}\text { Hemiparesia espástica } \\
\text { izquierda. }\end{array}$ \\
\hline
\end{tabular}


El infarto cerebral parece haber originado las convulsiones en los enfermos 1 y 3 , por la correlación anatomoclínica demostrada en ellos y la ausencia de otros factores etiológicos ${ }^{11}$, aun cuando en el paciente 3 la ECO reveló una pequeña hemorragia laminar parietal izquierda. En el caso 2 el sufrimiento fetal prenatal, el shock hipovolémico con probable isquemia cerebral y la presencia de hematomas subdurales, aparte del infarto cerebral mismo, pudieron haber sido factores que contribuyeron a producir las crisis. El origen multifactorial de las convulsiones neonatales ha sido observado en la práctica clínica y documentado en la literatura ${ }^{17,18}$. Desde este punto de vista, es necesario destacar que los 3 enfermos tuvieron LCR hemorrágico con punciones lumbares no traumáticas. Lo anterior, también puede ser una variable que favorezca las convulsiones, sobre todo si va acompanfada de otros factores ${ }^{11}$ que puedan indicar extravasación de sangre desde el sitio del infarto o pequeñas hemortagias subaracnoideas.

La etiologia del infarto es probablemente idiopática en los pacientes 1 y $3^{4,11}$ y de ori-

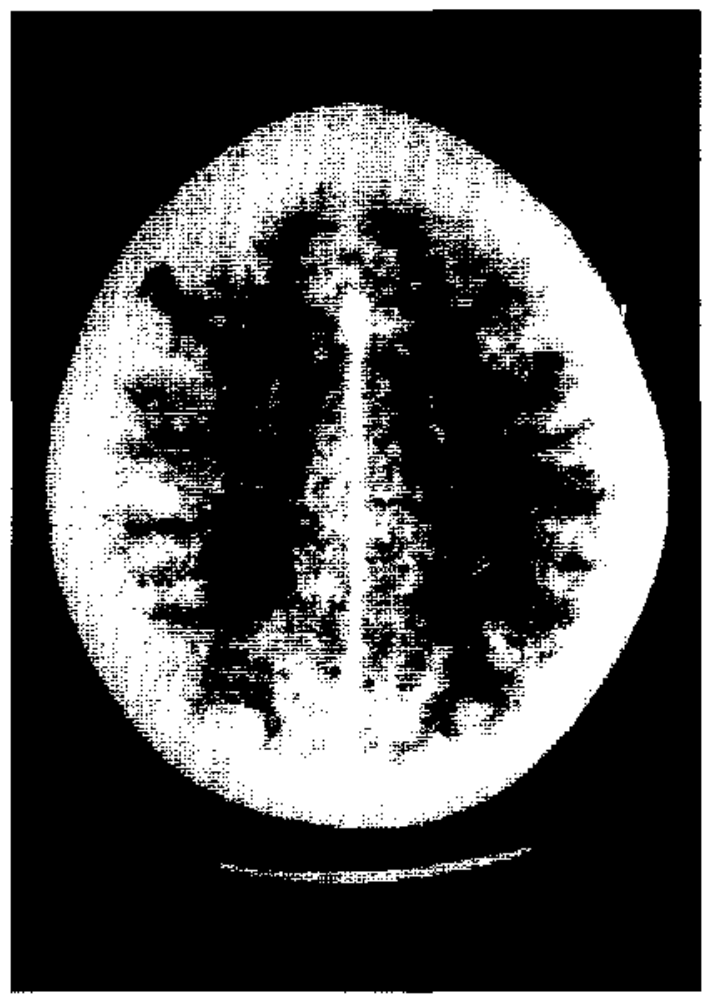

Figura 1: Tomografía axial computada del paciente 1. infarto cerebral derecho. gen isquémico en el caso $2^{6,9}$, pero variadas eventualidades han sido asociadas a infartos cerebrales en RNT con o sin convulsiones, documentadas clínica 0 aratomopatológicamente $5,6-9,12-13,19$.

Algunos infartos pueden originar leves signos e incluso pueden pasar inadvertidos ${ }^{5}$. No siempre hay convulsiones ${ }^{5,5,10,20}$; cuando existen pueden ser generalizadas (caso 2 ) $^{4,7,9}$ o focales como en los pacientes 1 y $3^{4,6,8,11-13}$. El examen físico, a diferencia de lo que ocurre en el niño mayor $y$ en el adulto, puede no dar en el primer momento signos locales, como aconteció en los 3 enfermos, sino, más bien, hipotonía e hiporreactividad, hecho que ha llamado la atención de diversos autores $4,5,8,9,11,14,19$. Sin embargo, el sitio de la lesión es decisivo para que ella se exprese clínicamente, dadas las características maduracionales del cerebro del $\mathrm{RN}^{20}$.

El electroencefalograma (EEG) parece ser un buen examen para sugerir una anormalidad fo$\mathrm{cal}^{4,8,9,13}$, tal como en los pacientes 2 y 3 , pero es probable que se necesiten algunos días

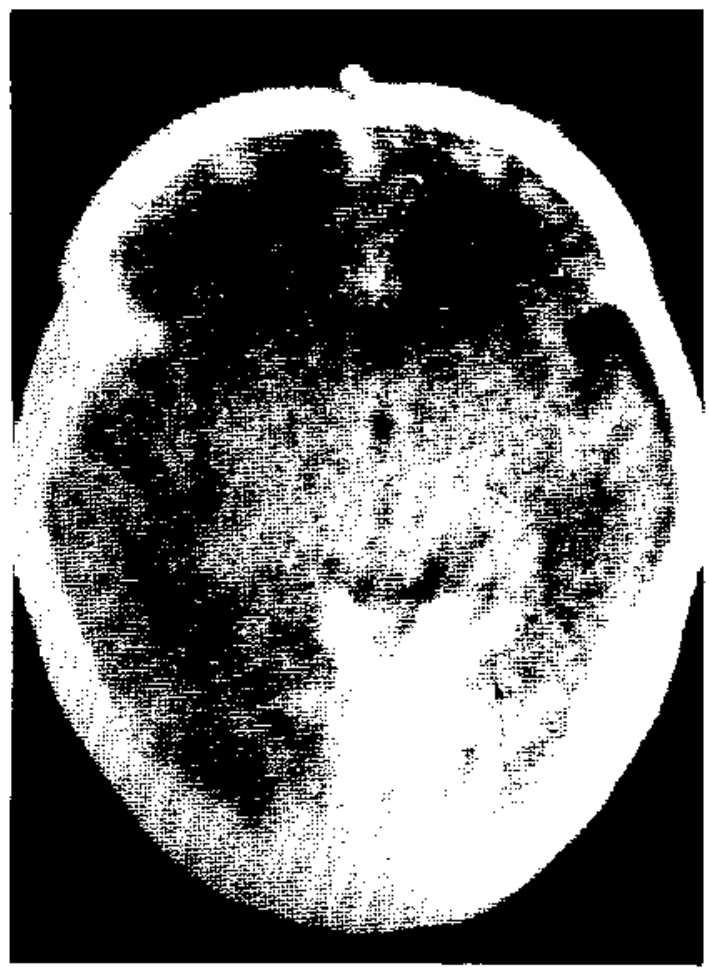

Figura 2: Tomografía axial computada del paciente 2, infarto cerebral derecho, hemorragia subaracnoidea bilateral. 
para que aparezca la evidencia. En efecto, en el caso 1, el EEG realizado el mismo día de las convulsiones resultó normal. En los otros dos enfermos, dicho examen fue practicado varios días después del inicio de las convulsiones y en ambos casos reveló alteraciones focales, indicadoras de daño orgánico cerebral.

La ecografía cerebral (ECO) es un proce. dimiento que no ha sido realizado sistemáticamente en los casos que se han publicado y su utilidad es controvertida ${ }^{B,}{ }^{9}$. En los 3 pacientes se practicó dicho examen dentro de las primeras 24 horas de iniciadas las convulsiones y no se demostró el infarto. En el enfermo 3, la ECO se repitió 4 días después y tampoco se observó la alteración vascular. Pareciera que este proce. dimiento es poco sensible para detectar el infarto en los primeros días ${ }^{8}$, pero realizado posteriormente podría ser de utilidad ${ }^{9}$.

El diagnóstico del infarto cerebral neonatal fue posible en los 3 enfermos sólo después de realizar la tomografía axial computada (TAC) en forma precoz en los casos 1 y 3 y nueve dí ts después de iniciadas las convulsiones en el caso 2. Se puede demostrar asi que éste es el examen más apropiado para confirmar la lesión vascular

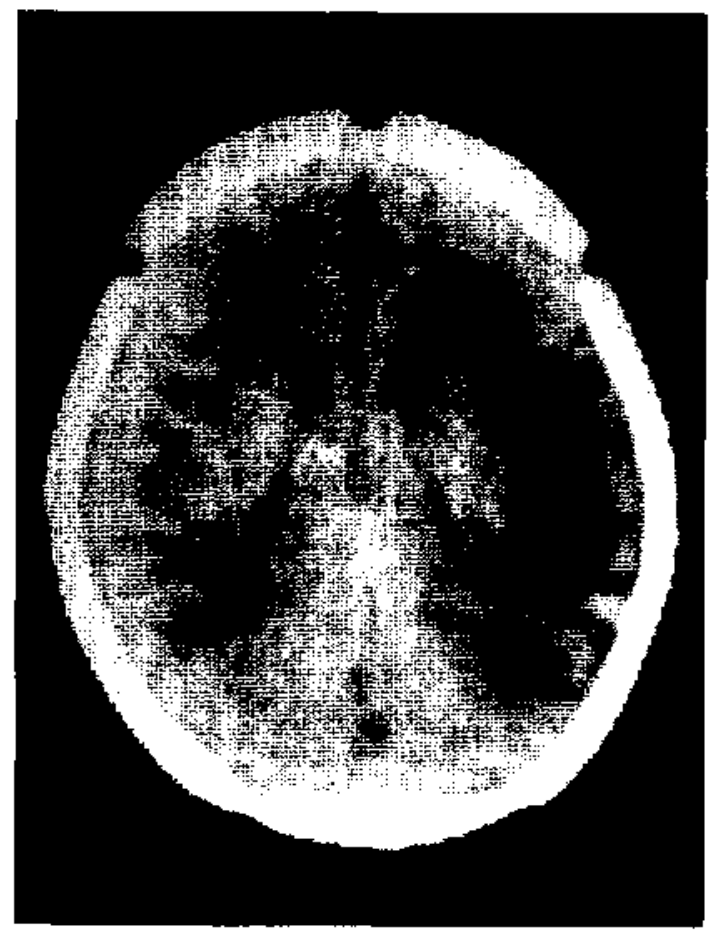

Figura 3. Tomografía axial computada del paciente 3 , infarto cerebral izquierdo. cerebral $4^{4}, 8-9,11,19$ y diagnosticarla sin tardanza $^{8,9}, 11,12$. Si bien es cierto que los RN no fallecen por el infarto mismo, sino por las causas que lo originan ${ }^{5}$, el diagnóstico correcto y precoz es importante para el pronóstico neurológico del nifno ${ }^{9}, 13$.

La evolución de nuestros pacientes en relación al desarrollo psicomotor ha sido, hasta el mo. ntento, normal, hecho concordante con seguimientos de menor ${ }^{7,8,11}$ y mayor duración ${ }^{4,13}$ que el nuestro. Siendo el infarto una lesión focal en un cerebro inmaduro, no es raro que la evolución sea favorable, pero se han des. crito atrasos globales del desarrollo ${ }^{10}$ y retardos del lenguaje ${ }^{4}$, complicaciones que pueden deberse al sitio de la leșión y a la causa que originó el daño vascular. La hemiparesia espástica constituye la complicación más frecuente ${ }^{4}, 7,8,11$ y se presentó en un enfermo de esta serie (caso 2). Hasta el momento no hemos detectado en los pacientes deficiencias de visión ${ }^{4}, 11$ y no sabemos qué ocurrirá en ellos con el aprendizaje de la lectoescritura. La epilepsia secundaria a in. fartos neonatales no ha sido detectada en la literatura, probablemente porque los seguitnientos son reducidos, pero es indudable que esta complicación no debería ser extraordinaria, dado el tipo de daño cerebral que puede originar el infarto $^{4,5,10}$, tal como ocurrió en uno de los pacientes.

De acuerdo a lo expuesto, pareciera necesario que en todo RNT con convulsiones se piense en la posibilidad de un infarto cerebral y se practiquen los exámenes destinados a diagnosticarlo. En este sentido, la TAC debería estar a disposición expedita de neonatólogos y neuropediatras.

\section{RESUMEN}

Se presentan 3 enfermos RNT con convulsiones e infarto cerebral, en dos no se logró identificar la causa, en el otro fue de origen isquémico. El examen neurológico y las ecografías encefálicas no parecen ser sensibles para detectar el infarto. Las crisis convulsivas y el EEG pueden sugerirlo, peco el diagnóstico definitivo requiere una tomografia axial computada del cerebro. El seguimiento de los pacientes por espacios de 1 año a 2 años 7 meses sugiere que el pronóstico es bueno en relación al desarrollo psicomotor, pero uno de los pacientes sufre de hemiparesia espástica y otro de epilepsia. 


\section{REFERENCIAS}

1. Holmes G.L.: Neonatal seizures. In: Pedley T.A., Meldrum B.S., eds.: Recent advances in epilepsy, London, England: Churchill Livingstone 1985; 20?-237.

1. Freeman J,M.: Neonatal seizures. ln: Dreifuss F.E., ed.: Pediatric Epileptology, Massachusetts, USA: John Wright $1983 ; 159-169$.

3. Fenichel G.M.: Neonatal Neurology, New York, USA: Churchill Livingstone $1980 ; 20-24$.

4. Levy S.R., Abroms I.F., Marshall P.C., Rosquete E.E.: Seizures and cerebral infarction in the fullterm Newborn. Ann Neurol 1985; 17 : 366-370.

5. Barmada M.A. Moosy J., Shuman R.M.: Cerebral infarcts with arterial oclusion in neonates. Ann Neurol $1979 ; 6: 495-502$

6. Ruff R.L., Shaw C.M., Beckwith J.B., Jozzo R. Y.: Ccrebral infartion complicating umbilical catheteIization. Ann Neurol 1978; 6: 85.

7. Amit M. Comfield P.R.: Neonatal polycythenia causing multiple cerebral infarcts. Arch Neurol 1980; $37: 109-110$.

8. Clancy R.R., Malin $S$, Iaraque D., Boumgart $S$.: Focal motor Seizures heralding strokes in meonate and infants (abstract). Ann Neurol 1983; 14: 370.

9. Hill A., Martin D.J., Daneman A., Fitz C.R.: Focal ischemic cerebral injury in the newborn: diagnosis by ultrasound and correlation with computed tomographic scan. Pediatrics 1983; 71: 790-793.

10. Ong B. Y., Ellison P.H., Browning $C$.: Intrauterine stroke in the neonate. Arch Neurol 1983; 40: 55-56.

11. Mantovani J.F., Gerber G.J.: "Idiopathie" neonatal cerebral infarction, Am J Dis Child 1984; 138 : $359-362$.

11. Chasnoff IJ., Bussey M.E., Savich R.. Stack C.M.: Perinatal cetebral inferction and matermal cocaine use. J Pediatr 1986; 108: 456-459

12. Trouner D.A., Mannino F.L.: Neurodevelopmental outcome after neonatal cerebrovascular accident. J Pediatr 1986; 108; 459-461.

14. Dulac O., Aubourg $P$., Plouin P.: Autres syndromes epileptiques du nouvcau-né. In: Roger J., Dravet C., Bureau $F_{\text {., }}$ Dreifuss F.E., Wolf P., eds.: Les Syndromes epileptiques de l'enfant et de l'adolescent, Montrougc. France: John Libbey Eurotext $1984 ; 23-31$.

15. Brosset Ph, Ronayette D., Tapie Ph. et al.: Les convulsions néonatales. Intéret d'un soore pronostique dans la conduit du traitement. Sem Hop Paris $1985 ; 61$ : 1832-1837.

16. Chiofalo $N$ : Epilepsias benignas del recién nacido, de] tiño y del adolescente. En: Devilat M., Ed.: Tallet Epilepsia 86, Sintiago, Chile: Fdición de Sociedad dc Neurologia, Psiquiatr ia y Neurocirugía de Chile 1986; 24-25.

17. Knouss T.A., Marshall R. E.: Seizures in a neonatat intensive care unit. Dev Med Child Neurol 1977; 19: 719-728.

18. Lombroseo C.T.: Neonatal Electaoncephalography. In: Niedemeyer E., Lopes da Silva F., Eds.: Elcctroencephatography, Baltimore, U.S.A. Urban and Schwarzenberg 1982; 599-637.

19. Roessmann U., Miller R.T.: Trombosis of the middle cerebral artery associated with birth trauma. Neurology 1980: 30: 889-892.

20. Pollack M.A., Llena J.F., Fleischmon A., Fish B.: Neonatal Brainstem infarction. Arch Neurol 1983; $40 \div 52-53$. 